This is an Accepted Manuscript of an article published by Taylor \& Francis in Basic and Applied Social Psychology on [date of publication tbc], available online: http://www.tandfonline.com/[Article DOI tbc].

\title{
Who do we trust on social policy interventions?
}

\author{
Magda Osman ${ }^{1}$, Norman Fenton ${ }^{1}$, Toby Pilditch², \\ David Lagnado², Martin Neil ${ }^{1}$
}

\begin{abstract}
Social policy interventions, such as nudges (behavioral change techniques), have gained significant traction globally. But what do the public think? Does the type of expert proposing a nudge influence the kinds of evaluations the public make about nudges? Three experiments investigated this by presenting a US ( $N=689)$ and UK sample $(N=978)$ with descriptions of nudges (genuine and fictitious) proposed by either scientists or the government. Overall, compared to opaque and fictitious nudges, transparent, and genuine nudges were judged more ethical and plausible, and scientists proposing them were judged more trustworthy than a government working group. Also, trust in fictitious interventions proposed by scientists was higher than in genuine interventions proposed by a government working group.
\end{abstract}

Keywords: Behavioral interventions, Nudge, Scientists vs. Government, Trust, social policy interventions

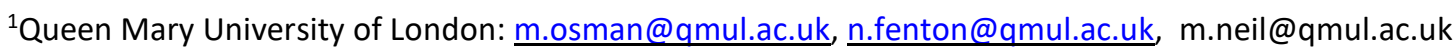

22University College London: t.pilditch@ucl.ac.uk, d.lagnado@ucl.ac.uk 


\section{Introduction}

Social scientific claims have become highly influential in the public domain, particularly recently in the context of social policy interventions. A dominant example of this is behavioral interventions, under the catch all term "nudges" (Thaler \& Sunstein, 2008), which have become highly popular decision-support methods used by governments to help in a wide range of areas such as health, personal finances, and general wellbeing (Curchin, 2017; Osman, 2016). The scientific claim, informed by psychological and behavioral economic research, is that to help people make better decisions regarding their lifestyle choices, and those that improve the welfare of the state, what is potentially effective is subtly changing the framing of the decision-making context, which makes prominent the option which maximizes long term future gains (Thaler \& Sunstein, 2008; Sunstein, 2016a). In essence the position adopted by Nudge enthusiasts is that poor social outcomes are often the result of poor decision-making, and in order to address this, behavioral interventions such as nudges can be used to reduce the likelihood of poor decisions being made in the first place (Curchin, 2017). This article aims to shed light on public opinion regarding social policy interventions, particularly with respect to the agent (Government, Scientist) that is proposing the intervention, and the extent to which trust is attributed to them. The key question of interest is the extent to which congruence between specific level of expertise (Government, Scientist) and the subject domain (behavioral interventions) impacts public trust in the agent for different types of interventions being proposed (plausibility of the interventions).

Nudges generally come in two types - opaque and transparent. Opaque nudges are purported to work covertly, so that the decision-maker has minimal awareness of the manipulation, and how it is designed to influence their behavior (e.g., defaults, rearrangement of choice alternatives, or using visual images to promote a positive or negative message). In contrast, transparent nudges explicitly identify what behavior needs changing and the reasons for this change (e.g., educational campaigns, clearer labelling on consumer products) (Felsen, Castelo, \& Reiner, 2013). In addition, nudges work for either the good of the individual (e.g., reducing calorie intake, increasing retirement funds, increasing exercise), referred to as pro-self nudges, or the good of the population (e.g., increasing organ 
donations, promoting recycling, home reducing energy usage), referred to as pro-social nudges (Hagman et al., 2015).

Considerable work has discussed and evaluated the effectiveness of different types of nudges (Benartzi et al, 2017; Berman \& Johnson, 2015; Johnson \& Goldstein, 2003; Marteau et al, 2011), how best to implement them (Grüne-Yanoff, \& Hertwig, 2016; Marteau, 2017), their ethical issues (Oliver, 2013; Osman, 2016; Sunstein, 2016a), and when they should be used to complement more conventional regulatory methods (e.g., mandates, taxes, bans, financial incentives) (House of Lords, 2011; Michie, van Stralen, \& West, 2011; Sunstein, 2014, 2016a).

While the predominate focus on studying nudges has been on establishing their efficacy, a new research focus in the social sciences has been directed towards examining public opinion towards them (Arad, \& Rubinstein, 2015; Felsen, Castelo, \& Reiner, 2013; Hagman et al., 2015; Hedlin, \& Sunstein, 2016; Jung \& Mellers, 2016; Reisch, \& Sunstein, 2016; Reisch, Sunstein \& Gwozdz, 2016; Sunstein, 2016b). Also, there has been work examining the publics' appetite for nudges over more conventional methods of social policy regulation (Mazzocchi et al., 2015; Sunstein, Reisch, \& Rauber, 2017). The findings show that the public is overwhelmingly more accepting of transparent over opaque nudges, though if told that opaque nudges are more effective, then there is marginal switching of favourable opinion towards opaque ones (Sunstein, 2016). Moreover, when presented with a choice between personal active choice or a behavioral intervention, the public opt for active choice, expressing an interesting in maintaining their own agency over their lifestyle choices (Arad, \& Rubinstein, 2015; Hedlin, \& Sunstein, 2016). There is also a stronger preference for pro-self (which impinge more on personal lifestyle choices) over pro-social nudges, although liberal political affiliation and higher empathic dispositions shift preferences towards pro-social nudges (Hagman et al., 2015; Jung \& Mellers, 2016; Tannenbaum, Fox, \& Rogers, 2015). While these findings provide some indication of how the public in the US, Europe, Africa, and Asia tend to consider nudges, the surveys predominately concern judgements of acceptance, approval, or preference. Few surveys consider the extent to which nudges are judged as restricting an individual's freedom, or whether they are judge to be effective (Arad, \& Rubinstein, 2015; Hagman et al., 2015), and none thus far have focused on examining the issue of trust, particularly with respect to the agent that is proposing the nudge. As such, the 
present set of experiments address, in depth, how the public judge the plausibility of behavioral interventions, their perceived transparency, their ethicality, and trust in the agent that is proposing them. In the experiments presented here, the public were required to make judgments about transparent and opaque nudges, in which the expert proposing the nudges was either a group of scientists with relevant expertise in the area, or a government working group with comparatively less relevant expertise (Experiment 1a, 1b; in Experiment 2 relevant expertise was matched in both groups). From the findings discussed here, public opinion is generally more favourable towards transparent compared to opaque nudges. By extension, in the set of experiments we conduct, we test the following hypothesis:

1. Hypothesis 1: Compared to actual opaque behavioral interventions, judgments of the perceived transparency of actual transparent behavioural interventions will be higher.

2. Hypothesis 2: Compared to opaque behavioral interventions, judgments of ethicality of transparent behavioural interventions will be higher.

3. Hypothesis 3: Compared to opaque behavioral interventions, judgments of plausibility of transparent behavioural interventions will be higher.

4. Hypothesis 4: Compared to opaque behavioral interventions, judgments of trust in the proposer of transparent behavioural interventions will be higher.

With respect to the first hypothesis, the vast majority of public surveys commonly explore public attitude towards transparent and opaque nudges. We extend this by examining the perceived transparency of the intervention that is designed to change behavior. The reason for including a question of this kind is as follows: we expect, given the critical differences in the social scientific definition between transparent and opaque nudges, that if the definitions are consistent with the public's perception of them, then as is outlined in Hypothesis 1, the more the public perceive a nudge as transparent the easier it is to identify how it changes behavior.

Taken together, the predicted direction of judgments outlined in Hypotheses 1-4 is based on the well-known Halo effect (Nisbett \& Wilson, 1977; Thorndike, 1920). A positive evaluation of transparent nudges should lead to positive attributions made regarding other dimensions which are related (perceived transparency, ethicality), as well as unrelated (plausibility). Also, as mentioned, there have been no public surveys on nudges that have explicitly examined 
trust in relation to the expert proposing the nudge (i.e. government, scientists). By expert we mean a speaker that is perceived to be capable of making correct and informed assertions (Pornpitakpan, 2004). By trust we mean the degree to which an audience perceives the assertions made by a communicator to be ones that the speaker considers valid (Hovland, Janis, \& Kelley, 1953).

Exploring these issue not only provides new insights in the domain of public attitudes on nudges, but also sheds light on growing academic interest in public trust of scientists. In particular, how people navigate and evaluate scientific communication (Strong, 2017). The way scientists communicate claims regarding matters of public interest has been investigated in areas such as climate change (Hmielowski,et al, 2014), genetically modified food (Lang, \& Hallman, 2005) and stem cell research (Critchley, 2008), to name but a few. Much of the work has considered the extent to which the public trusts scientific claims being made, and in some cases, how trust in scientists differs from the same claims being made by policy makers, political activists, and the media. Some work shows that scientists fare better than policy makers, government officials, and the media (Gauchat, 2012; IPSOS MORI, 2016; Marques, Critchley, \& Walshe, 2015). However, the opinion of scientists is guided by the public's own scientific knowledge regarding the topic of the scientific claims (Lewandowsky \& Oberauer, 2016; Sturgis, \& Allum, 2004), and their political affiliation; those leaning towards conservative political positions showing greater scepticism in scientists (Gauchat, 2012). Moreover, if scientists are seen to act in collaboration with politicians and industry then opinion drops (Critchley, 2008; Oreskes \& Conway, 2011). The latter finding suggests that the incentives that motivate scientific research also influences judgments of trust.

One of the many factors that can make communication persuasive to an audience (Petty \& Cacioppo, 1986; McGuire, 1978) is the source of the communication (Lewandowsky \& Oberauer, 2016; Lupia, 2013; Teeny, Briñol, \& Petty, 2016; for review see, Pornpitakpan, 2004). How does this apply in the context of scientific communication? Several studies have investigated the impact of credibility on the uptake of science communication online; there are now a vast range of online outlets from which scientific findings are communicated (Brossard, 2013). The evidence suggests that science communication is better recalled (McKnight \& Coronel, 2017), and is more appealing (Winter \& Krämer, 2012), when the topic 
and the expertise of the scientist are congruent. In other words, congruency effects based on the compatibility between the domain of expertise of the source (i.e. the expert) with the scientific topic make the communication more persuasive. The explanation here is that subject-area experts tend to have richer and more detailed knowledge of their own scientific topics of interest (Ericsson \& Lehmann, 1996). This means the information they communicate on the topic is more credible. Also, if the public has little or no expertise in the scientific topic, they will more likely put more trust in those judged to have highly relevant expertise in the topic they are discussing, especially if they communicate the information confidently (Sniezek \& Van Swol, 2001). Much the same is found in the area of advertising where the importance of congruency effects in source credibility has also been explored through what is known as the matching-hypothesis (Kamins, \& Gupta, 1994). When congruent, consumers will process both the endorser and the product more favourably (Amos, Holmes, \& Strutton, 2008; Fleck, Korchia, \& Le Roy, 2012).

In fact, congruency effects have a long established history in cognitive psychology, particularly in the domain of cognitive control (Notebaert, Gevers, Verbruggen, \& Liefooghe, 2006; Osman, 2014; Stroop, 1935). The congruency effect is well explained by the Monitoring Hypothesis (Botvinick, Braver, Barch, Carter, \& Cohen, 2001). This is based on a theoretical account of cognitive control which claims that there is top-down monitoring of information such that when a conflict is detected this prompts additional cognitive resources being allocated to the task at hand. A conflict, such as one between the scientific domain of expertise and the topic of communication, will lead to greater effort in processing which can then have damaging effects on judgments associated with the scientist and the topic.

Extending the Monitoring hypothesis to the focus of interest of the present set of experiments we consider the following: if there is a greater congruence between the domain of expertise and scientific topic area, then less cognitive effort will be spent processing the content of the scientific communication. Also, by the same token, more favourable judgments about the source and the content of the communication will be made. In the present set of experiments, the congruency between source and scientific communication was high in conditions where the expert (i.e. scientists researching in the topic of behavioural interventions) and the communication (i.e. presenting findings advocating behavioral 
interventions) closely matched. Congruency was low in conditions where the expert (i.e. a government working group that had no specific expertise in behavioural interventions) and the communication (i.e. presenting findings advocating behavioral interventions) was not specifically matched. Thus, in line with the monitoring hypothesis, and taken together with the evidence from research examining the congruency of domain of expertise and scientific topic, we test the following hypothesis:

5. Hypothesis 5: As a result of higher congruency between source and the topic of the science communication, the public will show higher judgments of trust for the high congruency conditions [scientists] compared to the low congruency conditions [a government group].

To further test the impact of the congruency manipulation we also manipulated the plausibility of the scientific details regarding the behavioral interventions. Two (i.e. exercise, personal finance) out of the five behavioral interventions described to participants have actually been implemented (i.e. plausible). To encourage more exercise nudges have been employed in stairwells to entice people to use them more (Åvitsland, Solbraa, \& Riiser, 2017; Kerr, Eves, \& Carroll, 2001; Marshall et al, 2002). To improving personal finance (e.g., investments, retirement funds) a pre-selected option is built into products to ease the burden of choice (Benartzi, et al, 2017; Drescher, Roosen, \& Marette, 2014). The remaining three nudges included in our experiments were completely fictitious and have not been implemented as behavioral interventions. However, they have attracted media attention because of their dubious nature as sources of behavioral change, and have featured in many discussions on scienceforum.com. On this point, the implausible inventions share a commonality with the plausible interventions, for the reason that serious doubts have been raised regarding their efficacy (for review see Lin, Osman, \& Aschroft, 2017).

As specified by the Monitoring Hypothesis (Notebaert, et al, 2006; Osman, 2014), when there is greater congruence, in this case between the domain of expertise and scientific topic area, then less cognitive effort will be spent processing critical information, i.e. the content of the scientific communication. Here we used the level of scrutiny regarding the plausibility of the behavioral interventions as a proxy for the amount of processing effort directed towards the 
content of the communication. So that we further test the impact of congruency in the following way:

6. Hypothesis 6: As a result of higher congruency between source and science communication, there will be higher judgments of trust in the High Congruency conditions [scientists proposing the interventions] compared to the Low Congruency conditions [a government working group], regardless of the plausibility of the behavioral intervention.

While public trust of scientific claims can be gained through high levels of source credibility, it can be easily eroded through revelations of financial ties to multinationals and conflicts of interest (Cho, et al, 2000; Dana \& Loewenstein, 2003; Elliott \& Resnik, 2014; Lewandowsky, Ecker, Seifert, Schwarz, \& Cook, 2012; McComas, 2008; Rosenstock \& Lee, 2002). Given that the present set of experiments are concerned with whether source credibility might influence trust in the communication of social science to support social policy interventions, we are also concerned with how easily trust can be eroded. Therefore, we examine the extent to which additional information about funding conditions that motivate the research carried out by the experts (i.e. scientists, government working group) differentially impacts judgments of trust. There is work to suggest that if trust in respected and credible institutions is high, then a revelation that can potential undermine that trust will lead to a more substantial drop in trust compared to institutions in which trust is already relatively lower (Löfstedt, 2003, 2005; McComas, 2008).

7. Hypothesis 7: Providing information regarding potential conflicts of interest in the motivatations behind proposing behavioral interventions will lead to a decrease in judgments of trust in the High Congruency conditions [scientists] compared to the Low Congruency conditions [a government working group].

\section{Experiment $1 \mathrm{a}$ and $1 \mathrm{~b}$}

\section{Methods}

\section{Design}


To investigate the various factors of interest we introduced the following critical between subject manipulations: 1) The agent proposing the social policy intervention (World leading scientists, Government working group, Control Condition - in which no information was provided); 2) The transparency of the intervention (transparent, opaque) which refers to the distinctions as defined in the nudge literature; 3 ) the presence of conflicts of interest in which information was presented indicating the underlying incentives for the scientists/government working group that motivated their proposing the interventions (Conflicts of Interest Absent [Experiment 1a], Conflict of Interest Present [Experiment 1b]).

Thus, Experiment 1a involved the following conditions: 1) High congruency condition (transparent) [interventions proposed by scientists]; 2) High congruency condition (opaque interventions) [interventions proposed by scientists]; 3) Low congruency condition (transparent) [interventions proposed by a government working group]; 4) Low congruency condition (opaque) [interventions proposed by a government working group]; 5) Control condition (transparent); 6) Control condition (opaque). In Experiment $1 \mathrm{~b}$ the same six conditions were presented, but the only difference was an additional piece of text that indicated the underlying incentives for the scientists/government working group that would have motivated the interventions they proposed; these details were only presented to the High Congruency and Low Congruency conditions, and not the control conditions.

Experiment $1 \mathrm{a}$ and $1 \mathrm{~b}$ presented participants with descriptions of five different behavioral interventions, and in each case participants were either presented with a transparent or opaque version of one of the interventions. Both experiments were run in November 2016 Two of the five behavioral interventions were based on genuine interventions that are currently being implemented presented in Table 1 . The three other interventions were completely fictitious. As mentioned, this was to examine the extent to which public opinion is sensitive to the plausibility of behavioral interventions that are proposed, and whether as a result of congruency effects, their higher estimation of the credibility of the scientists proposing the interventions overwhelms the assessment of the plausibility of the scientific claims being made. These are presented in Table 1. 
Table 1. Genuine and Fictitious behavioral interventions.

\begin{tabular}{|c|c|c|}
\hline Genuine Intervention & Transparent version & Opaque version \\
\hline Increasing fitness & posters on stairwells & art work on stairwells \\
\hline $\begin{array}{l}\text { Increasing retirement } \\
\text { savings }\end{array}$ & $\begin{array}{l}\text { a traffic light system (red, } \\
\text { amber, green) indicating } \\
\text { riskiness of retirement } \\
\text { products }\end{array}$ & $\begin{array}{l}\text { an automatic opt-out } \\
\text { system }\end{array}$ \\
\hline Fictitious Intervention & Transparent version & Opaque version \\
\hline $\begin{array}{l}\text { Increasing energy efficiency } \\
\text { by reducing hair-drier usage }\end{array}$ & Educational programme & $\begin{array}{l}\text { An automatic shut off } \\
\text { system after } 3 \text { mins }\end{array}$ \\
\hline $\begin{array}{l}\text { Decreasing the negative } \\
\text { (carcinogenic) impact of } \\
\text { drinking coffee by stirring } \\
\text { for } 60 \text { seconds }\end{array}$ & $\begin{array}{l}\text { Posters informing people to } \\
\text { stir coffee for } 60 \text { seconds }\end{array}$ & $\begin{array}{l}\text { Having café staff stir } \\
\text { coffee for } 60 \text { seconds }\end{array}$ \\
\hline $\begin{array}{l}\text { Increasing energy efficiency } \\
\text { by reducing number of right } \\
\text { hand turns made in vehicles } \\
\text { (left in USA) }\end{array}$ & $\begin{array}{l}\text { Signals on dashboards } \\
\text { instructing drivers to make } \\
\text { left turn }\end{array}$ & $\begin{array}{l}\text { Vehicles fitted with } \\
\text { steering wheels that } \\
\text { make it hard to perform } \\
\text { right turns }\end{array}$ \\
\hline
\end{tabular}

In sum, in the design of Experiment $1 \mathrm{a}$ and $1 \mathrm{~b}$ participants were randomly allocated to one of six conditions based on two critical manipulations adopting the following design: 2(Conflict of Interest: Absent [Experiment 1a], Present [Experiment 1b]) x 2 (Sample: US, UK) × 3 (Congruency of source and content: High congruency (i.e. Top Scientists), Low congruency (Government working group), Control) x 2 (Transparency: Transparent, Opaque) x 2 (Plausibility: High [Genuine interventions], Low [Fictitious interventions]). After they were presented with 5 demographic questions, participants were then provided descriptions of 5 behavioral interventions, the presentation of which was randomized for each. For the 5 behavioral interventions ( 2 genuine, 3 fictitious) all participants were required to respond to for judgment questions concerning: 1) the perceived transparency of the intervention designed to change behavior, 2) plausibility of behavioral interventions, 3) the ethicality of the interventions, and 4) trust in the agent that is proposing the interventions, also presented in random order. 


\section{Participants}

Experiment $1 \mathrm{a}$ and $1 \mathrm{~b}$ included US and UK samples (Experiment 1a: US $N=322$, UK $N=246$; Experiment 1b: US $N=367$, UK $N=368$ ) They were presented via Qualtrics and launched via Prolific Academic - a crowd sourcing system for participant recruitment worldwide. All participants were financially compensated for their time (90 cents). The eligible population size from which participants were recruited on Prolific academic was approximately 10,000 US citizens, and 9,000 UK citizens. We used this as a basis on which to determine our sample sizes. For a confidence interval of $99 \%$ with a margin of error of $5 \%$, the required sample size is 610-622 (Barlett, Kotrlik, \& Higgins, 2001). For both experiments ethics approval from QMUL college ethics board was granted under the project titled Ethical concerns around nudges, QMERC2014/54. Participants were presented with the questions online. Participants were given 4 probative questions regarding behavioral interventions but were first presented with 5 demographic questions (the responses to which are summarised in Table 2). With regards to the demographic questions, participants were asked to type their age in a text box [continuous measure], and provide details of their gender (Female [score 1], Male [score 2], Rather not say [Score 3]), along with their political affiliation (Left [Score 1], Centre [Score 2], Right [Score 3], Other [Score 4]), their educational background (High school [Score 1], diploma/foundation [Score 2], Bachelors degree [Score 3], Masters degree [Score 4], PhD [Score 5], Other [Score 6]), and religious orientation (Definitely religious [Score 1], Not sure [Score 2], Definitely not religious [Score 3]). These formed the basic question regarding demographics and social/cultural characteristics.

Table 2 Participants profile from Experiment $1 \mathrm{a}$ and $1 \mathrm{~b}$ combined, and Experiment 2.

\begin{tabular}{llll}
\hline Experiment & Experiment 1a \& 1b & Experiment 1a \& 1b & Experiment 2 \\
\hline Sample & US & UK & UK \\
$\begin{array}{l}\text { Total } \\
\text { participants }\end{array}$ & $\begin{array}{l}689 \text { (all US residents or } \\
\text { nationals, first language } \\
\text { English) }\end{array}$ & $\begin{array}{l}614 \quad \text { (all UK residents or } \\
\text { nationals, first language } \\
\text { English) }\end{array}$ & $\begin{array}{l}364 \quad \text { nationals, first language } \\
\text { English) }\end{array}$ \\
Females & $313(45 \%)$ & $337(55 \%)$ & $276(75 \%)$ \\
Age & $\begin{array}{l}\text { Mean } 32.66(S D=12.01) \\
\text { ranging from 18-74 }\end{array}$ & $\begin{array}{l}\text { Mean } 36.09(S D=12.73) \\
\text { ranging from } 18-72\end{array}$ & $\begin{array}{l}\text { Mean } 36.23(S D=11.06) \\
\text { ranging from } 18-72\end{array}$
\end{tabular}


Educational Mixed, 54.4\% qualified background with a degree (at bachelor degree and postgraduate level).

\section{Political affiliation}

Religion
Mixed, $58.4 \%$ qualified with a degree (at bachelor degree and postgraduate level).

45.3\% identifying themselves as left, $10.4 \%$ as centre, $17.8 \%$ as right, and $26.5 \%$ as other

$51 \%$ reported that they did not have one, $17.3 \%$ reported that they were not sure, $1 \%$ preferred not to say, and $30.8 \%$ reported that they had a religion
Mixed, $54.4 \%$ qualified with a degree (at bachelor degree and postgraduate level).

$32.1 \%$ identifying themselves as left, $13.7 \%$ as centre, $14.3 \%$ as right, and $39.8 \%$ as other

$53.1 \%$ reported that they did not have one, $8 \%$ reported that they were not sure, $1 \%$ preferred not to say, and $37.9 \%$ reported that they had a religion

\section{Procedure}

General instructions for all six conditions: After consenting to take part in the Experiment, all participants were provided with the following general instructions which were implemented for Experiment $1 \mathrm{a}$ and $1 \mathrm{~b}$. "This study examines attitudes towards Behaviour Change. In this study we are going to ask you a number of questions on the subject of the use of methods to change people's behavior. If you agree to take part in this research, you will be asked to give your views on the methods that have been proposed, and also answer some demographic questions. Your participation in this study will take about 14 minutes. This study has been approved by Queen Mary University Ethics board. Your participation is voluntary. Also, your participation in this research is confidential. In the event of publication of this research, no personally identifying information will be disclosed."

Instructions presented to the High congruent conditions in both Experiment $1 \mathrm{a} \& 1 \mathrm{~b}$; Participants were presented with the following: "For each of the following five scenarios that you will soon be presented with, the world's leading scientists in the relevant subject domain have developed a set of simple methods that adjust the way information is presented, so that it can help people to make better decisions. In each case the work was based on studies over more than 8 years, involved at least 3 Nobel prize winners and Professors working collaboratively from Universities including Harvard, MIT, Oxford and Cambridge. Each study 
was based on at least 120 peer-reviewed publications in the relevant leading academic journals. The reason for using these methods is to help improve people's behaviour, because in many day-to-day contexts people may not make a decision that is best for their own health, wealth, and their happiness".

In Experiment $1 b$, the following additional text, which was absent in Experiment $1 a$, directly followed after the above paragraph: "Depending on the success of the proposed methods, which requires an uptake of $20 \%$ of the target population, the Scientists will receive financial support for their continuing work on related activities for the next 5 years" This piece of text was also presented at the end of each of the five scenarios.

Instructions presented to the Low congruent conditions in both (Experiment 1a \& 1b): After the main instructions participants were then presented the following: "For each of the five following scenarios you will soon be presented with, a small working group of political activists with interests relevant to the subject matter have been developing a set of simple methods that adjust the way information is presented, so that it can help people to make better decisions. In each case the working groups were made up of politicians, members of the relevant special interest groups and members of the public, and for each case the recommendations were based on extended discussions with relevant stakeholders. The reason for using these methods in each scenario is to help improve people's behavior, because in many day-to-day contexts people may not make a decision that is best for their own health, wealth, and their happiness."

In Experiment $1 b$, the following additional text, which was absent in Experiment $1 \mathrm{a}$, directly followed after the above paragraph: "Depending on the success of the proposed methods, which requires an uptake of $20 \%$ of the target population, the Government Working Group will receive financial support for their continuing work on related activities for the next 5 years" This piece of text was also presented at the end of each of the five scenarios.

Instructions presented to the Control conditions (Experiment $1 \mathrm{a} \& 1 \mathrm{~b}$ ): The instructions presented after the main general instructions were "In the following pages, you will review 5 contexts and within each context you will answer a set of questions in relation to that 
context." Then for each description of the behavioural intervention, participants were presented with just those details (none of the additional text presented in the High Congruent and Low Congruent conditions) and then asked to respond to the four dependent measures (identification, ethicality, plausibility, trust - detailed below). Crucially for the trust question, participants in all Control conditions were presented with two versions. They had to respond to both for each of the five descriptions of the behavioural interventions. Imagine that a Government working group planned to recommend this method, to what extent do you trust that they have your best interests in mind? [Scale $1=1$ don't trust them at all $-9=1$ fully trust them]. Imagine that a group of Top Scientists planned to recommend this method, to what extent do you trust that they have your best interests in mind? [Scale $1=$ I don't trust them at all $-9=$ I fully trust them]. The reason for this was to provide a direct baseline comparison for responses to the trust questions presented in the High congruent and Low congruent conditions.

The general details of the scenarios presented in Table 3 were amended so that they were sensitive to the sample, i.e. National Health Service (NHS) for the UK sample was replaced with Health services for the US sample, and financial details were converted into \$ for the US sample, finally, the left/right hand steering for Fictitious scenario 3 was amended to be sample sensitive (UK = Right turn, US = left turn).

Table 3. Genuine and Fictitious scenarios.

\section{Transparent}

This piece of text appeared at the end of each scenario. There are two types of methods: Transparent and non-transparent.

A transparent method works in such a way that anyone can easily identify the actual method used to change their behavior, as well as easily identify how their behavior is changed by it.

A non-transparent method works in such a way that no one can identify the actual method used to change their behavior, and no one can identify how their behavior is changed by it.
Opaque

This piece of text appeared at the end of each scenario. There are two types of methods: Transparent and non-transparent.

A transparent method works in such a way that anyone can easily identify the actual method used to change their behavior, as well as easily identify how their behavior is changed by it.

A non-transparent method works in such a way that no one can identify the actual method used to change their behavior, and no one can identify how their behavior is changed by it. 
Genuine

1

In order to increase exercise, and increase peoples' fitness levels, people should climb between 20-25 flights of stairs each day in order to improve their aerobic power and in turn their cardiorespiratory fitness. It is estimated that doing this will increase individual life expectancy by 5 years, and if undertaken by each person not currently doing regular exercise, it would save the NHS $£ 1.2$ billion per year within 3 years.

METHOD: Stairwells now have posters with messages placed at strategic positions so as to encourage people to use stairs instead of lifts or escalators/elevators.

Genuine

In order to save more money for their retirement, people should pay more attention to the riskiness of their investment schemes, because in most cases people make poor financial decisions. By taking fewer risks, it is estimated that doing this will increase individual personal savings by $42 \%$, and if undertaken by each person that is not currently saving enough for their retirement the economic burden on social services would be reduced by $28 \%$ within a 5 year period.

METHOD: For any investment scheme that an individual plans to put their savings into (for retirement purposes), a traffic light system is presented to them to indicate which is the low risk and which is the high risk investment scheme.

Fictitious In order to reduce energy consumption in the 1 home, and save money on fuel bills, the most effective means is to limit the usage of hair dryers to 3 minutes. Compared to all other common home appliances, hair dryers use the most power (Watts) per hour, especially if they were bought before January 2016. It is estimated that if this measure was adopted across the world it would lead to a reduction of 30 tons of carbon emissions and would help most countries meet their carbon reduction emission targets within 20 years.

METHOD: An advertising campaign has been used to educate people as to the appropriate length of time that they should use hair dryers.

Fictitious In order to prevent any of the harmful effects of
In order to increase exercise, and increase peoples' fitness levels, people should climb between 20-25 flights of stairs each day in order to improve their aerobic power and in turn their cardiorespiratory fitness. It is estimated that doing this will increase individual life expectancy by 5 years, and if undertaken by each person not currently doing regular exercise it would save the NHS $£ 1.2$ billion per year within 3 years.

METHOD: Stairwells have art work place on each floor so as to encourage people to walk up the stairs to view the art work.

In order to save more money for their retirement people should pay more attention to the riskiness of their investment schemes, because in most cases people make poor financial decisions. By taking fewer risks, it is estimated that doing this will increase individual personal savings by $42 \%$, and if undertaken by each person that is not currently saving enough for their retirement the economic burden on social services would be reduced by $28 \%$ within a 5 year period.

METHOD: Banks and public service employers are now introducing default methods, so that the low risk investment fund is the default fund that people are automatically allocated to for any savings plan for their retirement.

In order to reduce energy consumption in the home, and save money on fuel bills, the most effective means is to limit the usage of hair dryers to 3 minutes. Compared to all other common home appliances, hair dryers use the most power (Watts) per hour, especially if they were bought before January 2016. It is estimated that if this measure was adopted across the world it would lead to a reduction of 30 tons of carbon emissions and would help most countries meet their carbon reduction emission targets within 20 years.

METHOD: Hairdryers will have automatic shutoff systems such that after 3 minutes of use, they will switch-off, and won't operate again until 10 minutes later.

In order to prevent any of the harmful effects of drinking coffee regularly, the most reliable way to do this is to continuously stir the coffee for 
approximately 60 seconds in order to prevent the release of chemicals (salicylates) that increase blood glucose levels and increase inflammation associated with diabetes and cardiovascular disease. It is estimated that doing this will increase individual life expectancy of regular coffee drinkers by 3 years, and if undertaken by each regular coffee drinker would save the NHS $£ 1$ billion per year within 5 years.

METHOD: The signage in cafes reminds you of the health benefits of stirring coffee for 60 seconds.

Fictitious In order to help reduce carbon emissions, and 3 improve fuel efficiency, all vehicles in the UK

should minimize making right turns wherever possible. Fuel consumption of all motor vehicles is substantially uneven with right turns consuming nearly twice as much fuel as left turns. It is estimated that if every driver in the UK reduced the number of right turns they make by $85 \%$ then within 5 years the UK will meet its carbon reduction emission targets.

METHOD: Vehicles now have signals on the dashboard that indicate the level of fuel consumed after each turn is performed.

approximately 60 seconds in order to prevent the release of chemicals (salicylates) that increase blood glucose levels and increase inflammation associated with diabetes and cardiovascular disease. It is estimated that doing this will increase individual life expectancy of regular coffee drinkers by 3 years, and if undertaken by each regular coffee drinker would save the NHS $£ 1$ billion per year within 5 years.

METHOD: The Defaulted option is that coffee served in cafes is stirred for you for 60 seconds.

In order to help reduce carbon emissions, and improve fuel efficiency, all vehicles in the UK should minimize making right turns wherever possible. Fuel consumption of all motor vehicles is substantially uneven with right turns consuming nearly twice as much fuel as left turns. It is estimated that if every driver in the UK reduced the number of right turns they make by $85 \%$ then within 5 years the UK will meet its carbon reduction emission targets.

METHOD: Vehicles are fitted with steering wheel devices that make it harder to complete a full right turn.

\section{Dependent Measures presented to every participant:}

For each scenario participants were asked the following four probative questions.

1) To what extent is it easy for you to identify HOW your behavior is going to be changed by the psychological method? [Scale $1=$ I cannot easily identify how my behavior is changed by the method to $100=$ I can easily identify how my behavior is changed by the method]. The mean responses were calculated separately for genuine and fictitious interventions.

2) To what extent do you think the proposed method is plausible? [Scale $1=$ not plausible at all $-100=$ completely plausible]. The mean responses were calculated separately for genuine and fictitious interventions. 
3) Do you think there are ethical issues concerning this method? [Response options $\mathrm{Yes} / \mathrm{No}$. The proportion of $\mathrm{y} / \mathrm{h}$ responses were coded separately for genuine and fictitious interventions.

4) Given that the $\{$ "Government working group"/"Academic working group" $\}$ plan to recommend this method, to what extent do you trust that they have your best interests in mind? [Scale $1=\mid$ don't trust them at all $-9=\mid$ fully trust them]. The mean responses were calculated separately for genuine and fictitious interventions.

Once participants had completed all questions for all five scenarios, the experiment was complete.

\section{Results}

Both Cohen's $d$ and partial $\eta^{2}$ are presented in the results section in Experiment $1(1 a, 1 b)$ and Experiment 2. Cohen (1988) provides a benchmark to define small (.1), medium (.3) and large (.5) effects where Cohen's $d$ is presented. Where partial $\eta^{2}$ effect sizes are presented we use the classification which specifies effects as small (.02), medium (.13) and large (.26) (Bakeman, 2005). As a consequence of the experimental design, our models include more than one IV, and only in a few planned comparisons did we compare against a control condition. Therefore, for these reasons, and to keep the continuity of reporting the results simple across comparisons, and erring on the side of caution, we use partial $\eta^{2}$ effect size classification, which in all comparisons appears to be more conservative that Cohen's $d$. 
Table 4.

Results of pairwise comparisons across Experiments 1a, Experiment 1b, and Experiment 2 in which the comparison statistics are presented ( $\eta^{2}$ and Cohen's $d$ ).

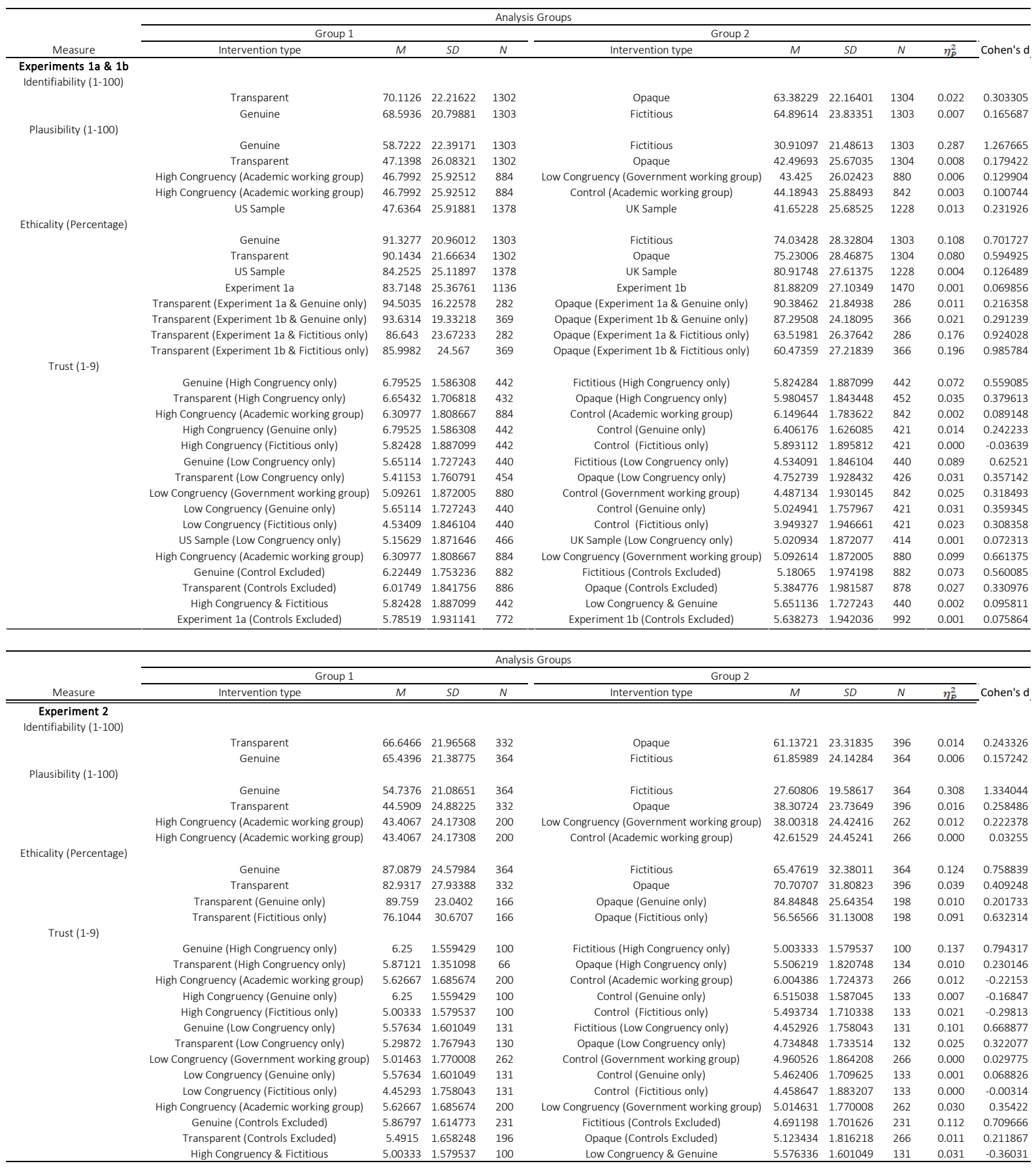

The results presented in Table 4 are the pairwise comparisons (for all Experiments) organised in the order they appear in the results section of the present paper, separated by dependent 
variable. The comparison statistics include a comprehensive list of key comparisons $\left(\eta_{P}^{2}\right.$ effect sizes and Cohen's $d$ ).

Judgments of perceived transparency: For these and all remaining analyses presented, no observations were excluded. To examine the impact of our manipulations on judgments regarding perceived transparency we conducted a 2(Conflict of Interest: Absent [Experiment 1a], Present [Experiment 1b]) $\times 2$ (Sample: US, UK) $\times 3$ (Congruency of source and content: High congruency (Scientists), Low congruency (Government working group), Control) x 2 (Transparency: Transparent, Opaque) x 2 (Plausibility: High [Genuine interventions], Low [Fictitious interventions]) analysis of variance, along with a series of pairwise comparisons. Overall, the analyses showed that there was a small effect such that for transparent interventions participants found it easier to identify how their behavior would be changed $(M$ $=70.113, S D=22.22, N=1302)$ as compared to opaque interventions $(M=63.38, S D=22.16$, $N=1304),\left(\eta_{P}^{2}=.02\right.$ [small], $d=.30$ [medium]). In addition, there was a very small effect suggesting that perceived transparency was judged higher for genuine $(M=68.59, S D=$ 20.80, $N=1303)$ compared to fictitious $(M=64.90, S D=23.83, N=1303)$ interventions, $\left(\eta_{P}^{2}=\right.$ .007 [very small], $d=.17$ [small]).

Judgments of plausibility: There was a large effect suggesting that genuine interventions $(M=$ $58.72, S D=22.39, N=1303)$ were judged more plausible than fictitious interventions $(M=$ 30.91, $S D=21.49, N=1303),\left(\eta_{P}^{2}=.29\right.$ [large] $d=1.27$ [large] $)$ t this indicates that our manipulation regarding the plausibility of the contexts was successful. There was a very small effect on plausibility judgments suggesting that they were impacted by transparency, with transparent interventions $(M=47.14, S D=26.08, N=1302)$ judged more plausible than opaque interventions $(M=42.50, S D=25.67, N=1304),\left(\eta_{P}^{2}=.008\right.$ [very small], $d=.17$ [small]). There was also a small effect suggesting that overall interventions were judged as more plausible in the High Congruent conditions $(M=46.80, S D=25.92, N=884)$, as compared to the Low Congruent conditions $(M=43.43, S D=26.02, N=880),\left(\eta_{P}^{2}=.006\right.$ [very small], $d=.13$ [small]). A very small effect indicated that interventions were also judged as more plausible in the High Congruent Conditions compared to Control conditions $(M=44.19$, $S D=25.92, N=842),\left(\eta_{P}^{2}=.003\right.$ [very small], $d=.10$ [small]). In addition, there was a small effect suggesting sample differences. The US sample gave higher overall plausibility 
judgments $(M=47.64, S D=25.92, N=1378)$ compared to the UK sample $(M=41.65, S D=$ 25.69, $N=1228),\left(\eta_{P}^{2}=.01\right.$ [small],$d=.23$ [small]).

Ethicality judgments: Ethicality judgments were based on a scoring of the percentage of interventions judged as ethical for genuine interventions and separately for fictitious interventions. Overall, a medium effect suggested that there was a higher percentage of favourable ethical judgments for genuine $(M=91.33, S D=20.96, N=1303)$ compared to fictitious $(M=74.03, S D=28.33, N=1303)$ interventions, $\left(\eta_{P}^{2}=.11\right.$ [small], $d=.70$ [large]), and small effect suggest that transparent interventions $(M=90.143, S D=21.67, N=1302)$ were judged as more ethical than opaque $(M=75.23, S D=28.47, N=1304),\left(\eta_{P}^{2}=.08\right.$ [small], $d=.59$ [large]). A very small effect indicated that interventions were generally considered more ethical by participants in the US $(M=84.25, S D=25.12, N=1378)$ than those in the UK $(M=80.92, S D=27.61, N=1228),\left(\eta_{P}^{2}=.004\right.$ [very small], $d=.13$ [small]). A very small effect indicated that ethicality judgments were impacted by manipulations regarding the details of the incentives (absent in Experiment 1a, present in Experiment $1 b)\left(\eta_{P}^{2}=.001\right.$ [very small], $d$ $=.07$ [small]). There was also a very small Congruency $\mathrm{x}$ transparency $\mathrm{x}$ type of intervention interaction, $\left(\eta_{P}^{2}=.001\right.$ [very small, $d=.22$ [small]). For genuine interventions, in the absence of conflicts of interest [Experiment 1a], there was a small effect $\left(\eta_{P}^{2}=.01\right.$ [small], $d=.22$ [small]) when comparing ethicality judgments for transparent $(M=94.50, S D=16.23, N=$ 282) and opaque versions $(M=90.38, S D=21.85, N=286)$. Similarly, in the presence of conflicts of interest [Experiment $1 \mathrm{~b}$ ] there was also a small effect $\left(\eta_{P}^{2}=.02\right.$ [small], $d=.30$ [medium]), ethical judgments were higher for transparent $(M=93.63, S D=19.33, N=369)$ compared to opaque versions $(M=87.30, S D=24.18, N=366)$. For fictitious interventions, there was a medium effect $\left(\eta_{P}^{2}=.18\right.$ [medium], $d=.92$ [large]), in the absence of conflicts of interest [Experiment 1a] ethicality judgments were higher for transparent $(M=86.64, S D=$ 23.67, $N=282)$ than opaque versions $(M=63.52, S D=26.37, N=286)$. There was a large effect $\left(\eta_{P}^{2}=.20\right.$ [large] $d=.99$ [large]) suggesting that in the presence of conflicts of interest [Experiment 1b] ethical judgments were higher for transparent $(M=86.00, S D=24.57, N=$ 369) compared to opaque interventions $(M=60.47, S D=27.22, N=366)$.

Judgments of Trust: When it comes to judgments of trust, separate comparisons were conducted between High Congruency conditions and Control conditions, and Low 
Congruency and Control conditions. Recall that for the Control conditions all participants were presented trust judgments for each type of expert (i.e. Scientists, Government working group) that could have been involved in proposing the behavioral interventions.

For trust judgments there was a small effect indicated that in the High Congruency conditions, trust was overall higher for genuine $(M=6.80, S D=1.59, N=442)$ compared to fictitious $(M=5.82, S D=1.89, N=442$ ) behavioral interventions (left-hand pair of bars, lefthand facet, Fig. 1), $\left(\eta_{P}^{2}=.07\right.$ [small], $d=.56$ [large]). There was also a small effect suggesting that there was greater trust for transparent $(M=6.65, S D=1.71, N=432)$ compared to opaque $(M=5.98, S D=1.84, N=452)$ interventions, $\left(\eta_{P}^{2}=.04\right.$ [small], $d=.38$ [medium]).

Fig. 1. Judgments of trust in interventions, split by source (facet), congruency manipulation (within-facet pairs) and genuine (white bars) vs fictitious (grey bars) nature. Error bars reflect standard error.

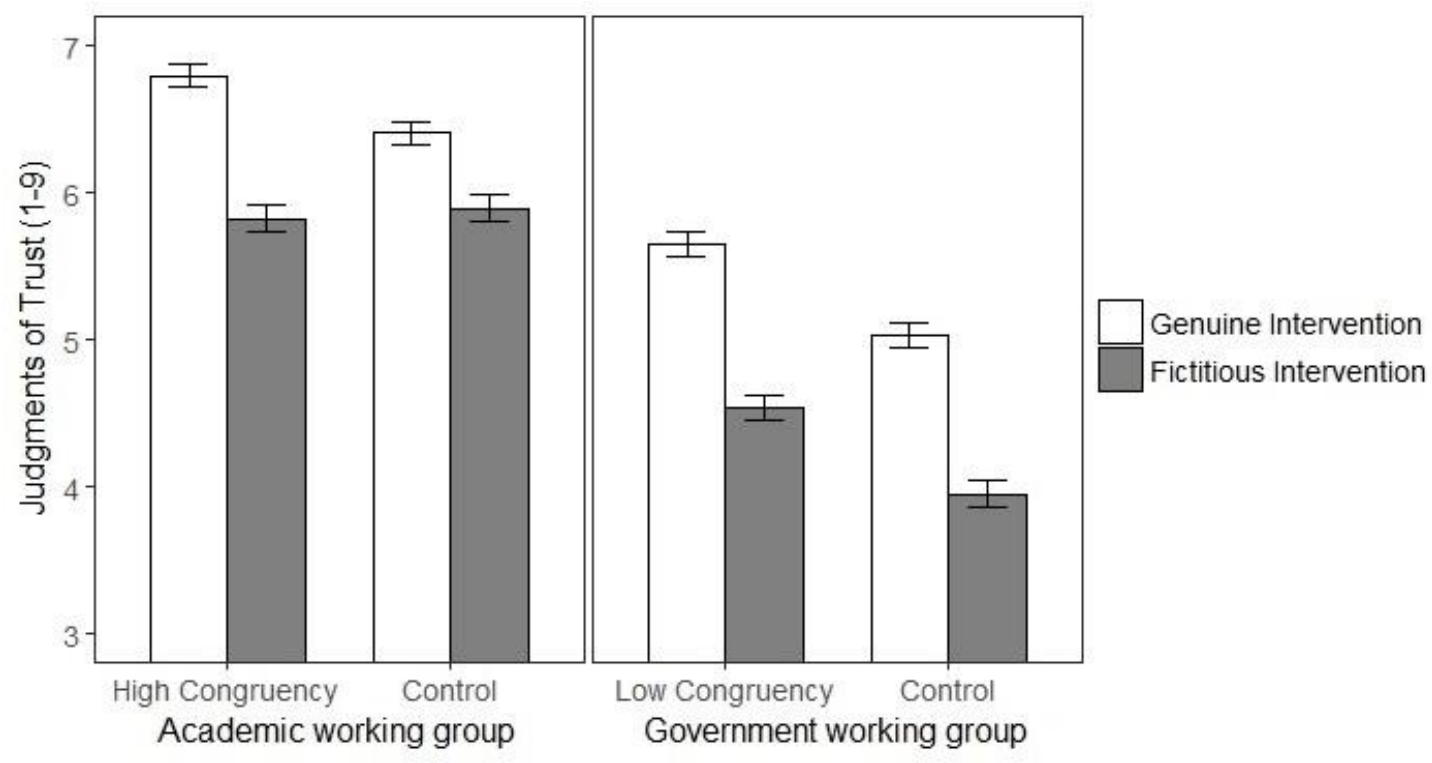

There was a very small effect suggesting that the congruency manipulation affected judgments of trust for genuine and fictitious interventions when comparing the High Congruency Conditions with the Control Conditions (High Congruency, $M=6.31, S D=1.81, N$ $=884$; Control Condition, $M=6.15, S D=1.78, N=842),\left(\eta_{P}^{2}=.002\right.$ [very small], $d=.09$ [small]). A small effect revealed that for genuine interventions (white bars, left-hand facet, Fig. 1), those in the High Congruency conditions judged scientists higher $(M=6.80, S D=1.59$, 
$N=442)$ than in the Control conditions $(M=5.41, S D=1.63, N=421),\left(\eta_{P}^{2}=.01\right.$ [small], $d=$ $.24[$ small]).

A medium effect revealed that trust judgments in the Low Congruency conditions were overall higher for genuine $(M=5.65, S D=1.73, N=440)$ compared to fictitious $(M=4.53, S D$ $=1.85, N=440$ ) behavioral interventions (left-hand pair of bars, right-hand facet, Fig. 1$),\left(\eta_{P}^{2}\right.$ $=.10$ [small], $d=.62$ [large]). There was a small effect revealing that there was greater trust for transparent $(M=5.41, S D=1.76, N=454)$ compared to opaque $(M=4.75, S D=1.93, N=$ 426) interventions, $\left(\eta_{P}^{2}=.03\right.$ [small], $d=.36$ [medium]).

A small effect showed that the congruency manipulation affected judgments of trust suggesting that overall trust judgments were higher in the Low Congruency conditions $(M=$ 5.09, $S D=1.87, N=880)$ compared to the Control conditions $(M=4.49, S D=1.93, N=842)$, $\left(\eta_{P}^{2}=.03\right.$ [small], $d=.32$ [medium]). Small effects revealed higher trust in the Low Congruency conditions over Control conditions carried through to both genuine (white bars, right-hand facet, Fig. $1 ;\left(\eta_{P}^{2}=.03\right.$ [small], $d=.36$ [medium])), and fictitious (grey bars, righthand facet, Fig. $1 ;\left(\eta_{P}^{2}=.02\right.$ [small], $d=.31$ [medium])) interventions. A very small sample difference was found in that the US sample gave higher ratings of trust in the Low Congruency conditions $(M=5.16, S D=1.87, N=466)$ compared to the UK sample $(M=5.02$, $S D=1.87, N=414),\left(\eta_{P}^{2}=.001\right.$ [very small], $d=.07$ [small]). On closer inspection, judgments of trust appeared to be higher for High Congruent conditions (left-hand pair of bars, left-hand facet, Fig. 1) compared to Low Congruency conditions (left-hand pair of bars, right-hand facet, Fig. 1), irrespective of whether the interventions were genuine or fictitious. To explore this, we directly compared judgments of trust between the High and Low Congruency conditions.

First of all, a small effect showed that trust (regardless of congruency) was higher overall for genuine $(M=6.22, S D=1.75, N=882)$ compared to fictitious interventions $(M=5.18, S D=$ 1.97, $N=882),\left(\eta_{P}^{2}=.07\right.$ [small], $d=.56$ [large]). A small effect also indicated that there was also greater trust for transparent $(M=6.02, S D=1.84, N=886)$ compared to opaque $(M=$ 5.38, $S D=1.98, N=878)$ interventions, $\left(\eta_{P}^{2}=.03\right.$ [small], $d=.33$ [medium]). Regardless of 
whether the intervention was genuine or fictitious, overall there was a medium effect showed that there were higher judgments of trust in High Congruency conditions $(M=6.31$, $S D=1.81, N=884)$ than Low Congruency conditions $(M=5.09, S D=1.87, N=880),\left(\eta_{P}^{2}=.10\right.$ [small], $d=.66$ [large])). To explore just how much trust in High Congruency conditions was greater than Low Congruency conditions, judgments for trust in High Congruency conditions for fictitious interventions only (left-hand grey bar, left-hand facet, Fig. 1; $M=5.82, S D=$ 1.89, $N=442$ ) were compared with judgments for trust in the Low Congruency conditions for genuine interventions only (left-hand white bar, right-hand facet, Fig. $1 ; M=5.65, S D=1.73$, $N=440$ ), showing a very small effect on trust judgments such that they were higher for High Congruency conditions in this comparison, $\left(\eta_{P}^{2}=.002\right.$ [very small], $d=.10$ [small]). Lastly, there was a very small effect showing that judgments of trust (collapsed across High and Low Congruency conditions) were also impacted by manipulations regarding details of the incentives (absent in Experiment 1a, present in Experiment 1b). More precisely, trust was judged to be higher when incentive details were absent (Experiment $1 \mathrm{a} ; M=5.79, S D=1.93$, $N=772$ ) than present (Experiment $1 b ; M=5.64, S D=1.94, N=992),\left(\eta_{P}^{2}=.001\right.$ [very small], $d=.07$ [small]). There were very small effects suggesting that when comparing the absence $(M=5.19, S D=1.60, N=197)$ and presence $(M=5.02, S D=1.60, N=123)$ of conflicts of interest on trust for High Congruency conditions, trust was lower in the presence of conflicts of interest, $\left(\eta_{P}^{2}=.003\right.$ [very small], $d=.11$ [small]). The same pattern was found for Low Congruency conditions, $\left(\eta_{P}^{2}=.002\right.$ [very small], $d=.17$ [small]).

Regression analyses conducted on the question probing trust in High and Low Congruency conditions separately for genuine and fictitious behavioral interventions revealed an association with Congruency, transparency, age and political affiliation, with an $\mathrm{R}^{2}$ ranging between .07 to .13. For genuine interventions in the Low Congruency conditions, judgments of trust decreased by .01 on the scale as age increased (decreased by .04 for fictitious interventions) and decreased by .20 on the scale the more conservative leaning participants were (decreased by .14 for fictitious interventions). A similar pattern was found for genuine interventions for High Congruency conditions, judgments of trust decreased by .01 on the scale as age increased (decreased by .03 for fictitious interventions) and decreased by .11 on the scale the more conservative leaning participants were (decreased by .21 for fictitious interventions). 


\section{Experiment 2}

One of the critical manipulations introduced in Experiment $1 \mathrm{a}$ and $1 \mathrm{~b}$ was the degree of congruency between the domain of expertise (scientist, government working group) and the behavioral interventions being proposed. In essence to ensure we found congruency effects, which indeed we found in support of Hypothesis 4, 5 and 6, participants in the High Congruency conditions were told that the agents were Top Scientists from prestigious universities and those in the Low Congruency conditions were told that the agents were a combination of special interest groups, members of the public and other stakeholders. Thus, the High and Low congruency conditions varied not only in terms of the compatibility between relevant expertise and the domain of science communication, but also the level of prestige associated with the expert. This may have contributed to the effects reported in Experiment $1 \mathrm{a}$ and $1 \mathrm{~b}$. To examine the robustness of the congruency effects, and to make the High and Low congruency conditions more directly comparable, we replicated Experiment $1 \mathrm{~b}$, but with minor changes to the instructions.

\section{Methods}

\section{Design}

To replicate the findings, and to address concerns regarding the potential confound of prestige with congruency effects, some minor changes were made to the instructions, but other than that the design of Experiment 2 was the same as Experiment $1 b$.

The critical difference was the change in instructions presented to the High and Low Congruency conditions. The text presented in Experiment $1 \mathrm{a}$ and $1 \mathrm{~b}$ for the High Congruency conditions "In each case the work was based on studies over more than 8 years, involved at least 3 Nobel prize winners and Professors working collaboratively from Universities including Harvard, MIT, Oxford and Cambridge. Each study was based on at least 120 peer-reviewed publications in the relevant leading academic journals." was replaced with "In each case, the Academic Working Group was made up of scientists with special knowledge and interest in subjects related to the interventions". The text presented in Experiment $1 \mathrm{a}$ and $1 \mathrm{~b}$ for the Low Congruency conditions "In each case the working groups were made up of politicians, 
members of the relevant special interest groups and members of the public, and for each case the recommendations were based on extended discussions with relevant stakeholders" was replaced with "In each case, the Government working group was made up of politicians and stakeholders with special knowledge and interest in subjects related to the interventions". Thus, the critical difference between the two conditions was the expertise, and prestige as well as the relative fit between expertise and the domain of the science communication.

Experiment 2 was run in January 2018. In Experiment 2 participants (364 UK residents or nationals, first language English (see Table 2)) and were randomly allocated to one of six conditions based on two critical manipulations adopting the following design: 3 (Congruency of source and content: High congruency (i.e. a scientific working group), Low congruency (a government working group), Control) x 2 (Transparency: Transparent, Opaque) $\times 2$ (Plausibility: High [Genuine interventions], Low [Fictitious interventions]). After they were presented with 5 demographic questions, participants were then provided descriptions of 5 behavioral interventions, the presentation of which was randomized for each. For the 5 behavioral interventions ( 2 genuine, 3 fictitious) all participants were required to respond to for judgment questions concerning: 1) the ease by which the way in which interventions changed behavior could be identified, 2) plausibility of behavioral interventions, 3) the ethicality of the interventions, and 4) trust in the agent that is proposing the interventions, also presented in random order.

\section{Results}

For all analyses presented, as in the analysis of Experiments $1 \mathrm{a}$ and $1 \mathrm{~b}$, no observations were excluded. To examine the impact of our manipulations on each DV, we conducted a 3 (Congruency of source and content: High congruency (Academic Working group), Low congruency (Government working group), Control) x 2 (Transparency: Transparent, Opaque) x 2 (Plausibility: High [Genuine interventions], Low [Fictitious interventions]) analysis of variance, along with a series of pairwise comparisons.

Judgments of perceived transparency: Overall, the analyses showed a small effect suggesting that perceived transparency was higher for transparent interventions $(M=66.647, S D=$ 
21.97, $N=332)$ than opaque interventions $(M=61.14, S D=23.32, N=396),\left(\eta_{P}^{2}=.01\right.$ [small], $d=.24$ [small]), replicating Experiments $1 \mathrm{a}$ and $1 \mathrm{~b}$. There was a very small effect indicating that the way in which behavior would change through the behavioural interventions was perceived as higher for genuine $(M=65.44, S D=21.39, N=364)$ as compared to fictitious ( $M$ $=61.86, S D=24.14, N=364)$ interventions, $\left(\eta_{P}^{2}=.006\right.$ [very small], $d=.16$ [small]).

Judgments of plausibility: In line with Experiments $1 \mathrm{a}$ and $1 \mathrm{~b}$, we found a large effect such that genuine interventions $(M=54.74, S D=21.09, N=364)$ were judged more plausible than fictitious interventions $(M=27.61, S D=19.59, N=364),\left(\eta_{P}^{2}=.31\right.$ [large], $d=1.33$ [large). There was also a small effect showing that plausibility judgments were impacted by transparency, with transparent interventions $(M=44.59, S D=24.88, N=332)$ judged more plausible than opaque interventions $(M=38.31, S D=23.74, N=396),\left(\eta_{P}^{2}=.02\right.$ [small], $d=$ .26 [small]). Also, there was a small effect revealing that interventions were judged as more plausible in the High Congruent conditions $(M=43.41, S D=24.17, N=200)$, as compared to the Low Congruent conditions $(M=38, S D=24.42, N=262),\left(\eta_{P}^{2}=.01\right.$ [small], $d=.22$ [small]).

Ethicality judgments: Ethicality judgments were (as in Experiments $1 \mathrm{a}$ and $1 \mathrm{~b}$ ) based on a scoring of the percentage of interventions judged as ethical for genuine interventions and separately for fictitious interventions. Overall, there was a medium effect suggesting more favourable ethical judgments for genuine $(M=87.09, S D=24.58, N=364)$ compared to fictitious $(M=65.48, S D=32.38, N=364)$ interventions, $\left(\eta_{P}^{2}=.12\right.$ [medium], $d=.76$ [large]). A small effect suggested that transparent interventions $(M=82.93, S D=27.93, N=332)$ were judged more ethical than opaque $(M=70.71, S D=31.81, N=396),\left(\eta_{P}^{2}=.04\right.$ [small], $d=.41$ [medium]). For genuine interventions, a small effect suggested that when comparing ethicality judgments for transparent $(M=89.76, S D=23.04, N=166)$ and opaque versions $(M=84.85, S D=25.64, N=198)$, the former was judged more ethical, $\left(\eta_{P}^{2}=.01\right.$ [small], $d=$ .20 [small]). Lastly, a small to medium effect for fictitious interventions, suggested that ethicality judgments were also higher for transparent $(M=76.1, S D=30.67, N=166)$ than opaque versions $(M=56.57, S D=31.13, N=198),\left(\eta_{P}^{2}=.09\right.$ [small], $d=.63$ [large]). 
Judgments of Trust: Following the protocol and rationale set out in the analysis of Experiments $1 \mathrm{a}$ and $1 \mathrm{~b}$, separate analyses were conducted comparing those in the High Congruency condition with the Control condition, and the Low Congruency Condition with the Control condition. For the Control conditions, all participants were asked general trust judgments of experts (i.e. Academic working group, Government working group) that could have been involved in proposing the behavioral interventions. A medium effect for trust judgments in High Congruency conditions suggested that trust was once again higher for genuine $(M=6.25, S D=1.56, N=100)$ compared to fictitious $(M=5.00, S D=1.58, N=100)$ interventions (left-hand pair of bars, left-hand facet, Fig. 2), $\left(\eta_{P}^{2}=.14\right.$ [medium], $d=.79$ [large]). In further replication of Experiments $1 \mathrm{a}$ and $1 \mathrm{~b}$, there was small effect showing that there was greater trust in High Congruency conditions for transparent $(M=5.87, S D=1.35, N$ = 66) compared to opaque $(M=5.51, S D=1.82, N=134)$ interventions, $\left(\eta_{P}^{2}=.01\right.$ [small], $d=$ .23 [small]).

A small effect suggested that the congruency manipulation also impacted judgments of trust for genuine and fictitious interventions when comparing High Congruency $(M=5.63, S D=$ 1.69, $N=200)$ to Control conditions $(M=6.00, S D=1.72, N=266),\left(\eta_{P}^{2}=.01\right.$ [small], $d=.22$ [small]). Very small differences were found between High Congruency conditions and Control conditions when conducing comparisons for genuine interventions only (white bars, left-hand facet, Fig. 2), $\left(\eta_{P}^{2}=.007\right.$ [very small], $d=.17$ [small]), and again for fictitious interventions only (grey bars, left-hand facet, Fig. 2), $\left(\eta_{P}^{2}=.02\right.$ [small], $d=.30$ [medium]). 
Fig. 2. Judgments of trust in interventions, split by source (facet), congruency manipulation (within-facet pairs) and genuine (white bars) vs fictitious (grey bars) nature. Error bars reflect standard error.

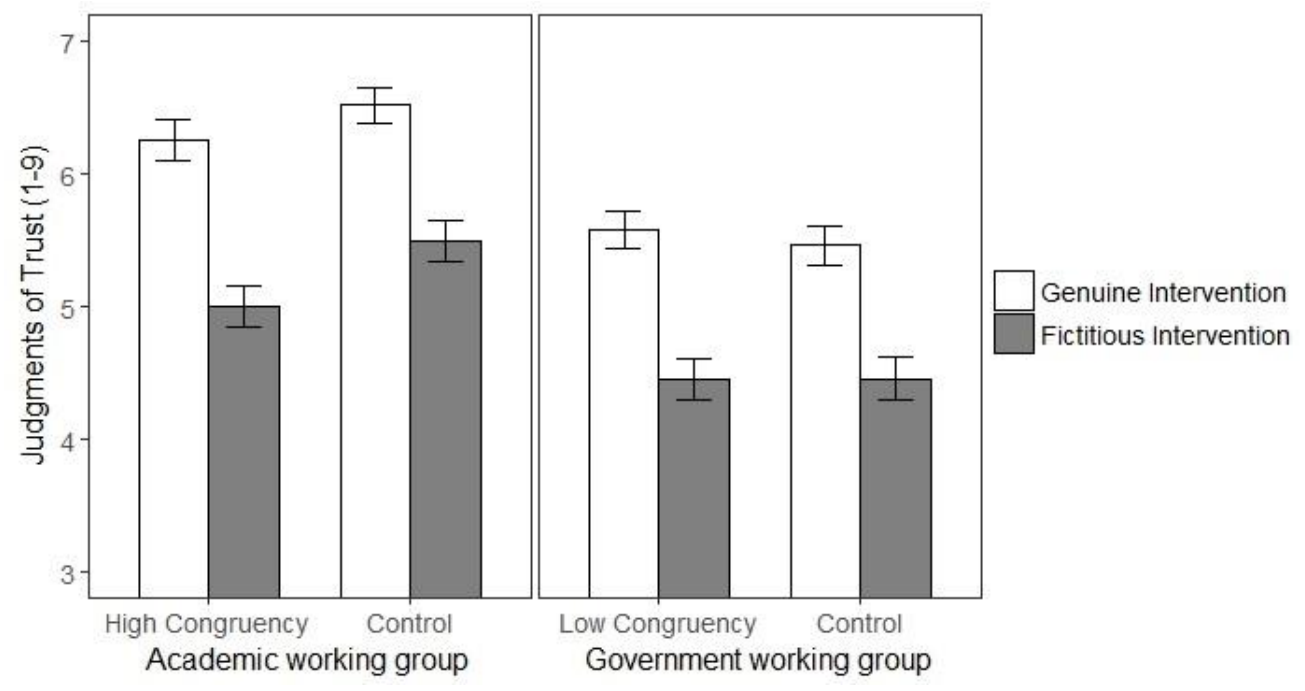

As in Experiments $1 a$ and $1 b$, the medium effect of trust in the Low Congruency conditions suggested that trust was higher for genuine $(M=5.58, S D=1.60, N=131)$ compared to fictitious $(M=4.45, S D=1.76, N=131$ ) interventions (left-hand pair of bars, right-hand facet, Fig. 2), $\left(\eta_{P}^{2}=.10\right.$ [medium], $d=.67$ [large]). There was also a small effect of trust in the Low Congruency conditions indicating that trust was higher when proposing transparent $(M=$ $5.30, S D=1.77, N=130)$ compared to opaque $(M=4.73, S D=1.73, N=132)$ interventions, $\left(\eta_{P}^{2}=.03[\right.$ small], $d=.32[$ medium] $)$.

Consistent with the pattern of findings in Experiment $1 a$ and $1 b$, in Experiment 2 there was a medium effect of Trust in the type of intervention, suggesting that trust was higher in genuine $(M=5.87, S D=1.61, N=231)$ versus fictitious interventions $(M=4.69, S D=1.70, N$ $=231$ ) regardless of congruency, $\left(\eta_{P}^{2}=.11\right.$ [medium], $d=.71$ [large]). Similarly, a small effect indicated that trust was higher in experts when proposing transparent $(M=5.49, S D=1.66, N$ $=196)$ as compared to opaque $(M=5.12, S D=1.82, N=266)$ interventions, $\left(\eta_{P}^{2}=.01\right.$ [small], $d=.21$ [small]). Also, in line with Experiments $1 \mathrm{a}$ and $1 \mathrm{~b}$, judgments of trust once again appeared to be higher for High Congruent conditions (left-hand pair of bars, left-hand facet, Fig. 2) compared to Low Congruency conditions (left-hand pair of bars, right-hand facet, Fig. 2). A small effect indicated that (irrespective of the genuine or fictitious nature of 
interventions) High Congruency conditions $(M=5.62, S D=1.69, N=200)$ again showed higher judgments of trust than the Low Congruency conditions $(M=5.01, S D=1.77, N=262)$, $\left(\eta_{P}^{2}=.03[\right.$ small], $d=.35[$ medium $])$.

Adopting the same comparison as that conducted in Experiment 1, to examine just how much trust is attributed in High Congruency conditions compared to Low Congruency conditions, judgments for trust in High Congruency conditions for fictitious interventions only (left-hand grey bar, left-hand facet, Fig. 2; $M=5.00, S D=1.58, N=100$ ) were compared with judgments for trust in the Low Congruency conditions for genuine interventions only (lefthand white bar, right-hand facet, Fig. $2 ; M=5.58, S D=1.60, N=131$ ). This time the pattern suggested that, unlike Experiment 1, in Experiment 2, a small effect on trust judgments revealed that they were higher for Low Congruency conditions in this comparison, $\left(\eta_{P}^{2}=.03\right.$ [small], $d=.36$ [medium]).

\section{General Discussion and Conclusions}

The current set of experiments were designed to give a richer profile of public opinion regarding nudge type behavioral interventions, and to extend the current work in this field. We tested 7 hypotheses, 3 of which were generated from the Monitoring framework (Botvinick, et al, 2001). In summary, we found evidence in line with Hypotheses 1-4. For all four dependent measures (perceived transparency, plausibility, ethicality, trust) in all three experiments the public consistently made more favourable judgments for transparent (small effects), and genuine interventions (small to large effects) as compared with opaque and fictitious interventions. The positive response to transparent behavioral interventions is compatible with recent public survey studies examining the level of approval of different types of nudges (Felsen, et al., 2013; Jung \& Mellers, 2016; Reisch, \& Sunstein, 2016; Reisch, et al., 2016; Sunstein, 2016b). The evidence that genuine over fictitious interventions were considered more favorably, is a novel finding. It indicates that the public clearly show sensitivity to the plausibility of nudges when making their own evaluations of them, especially when it comes to how much they trust them, and how ethical they consider them to be. 
Hypotheses 5-7 concerned the congruency manipulation and its impact on judgments of trust. Recall, that this measure assessed the extent to which the public judged the extent to which they trusted that the agent proposing the intervention had the public's interest. Experiments $1 \mathrm{a}, 1 \mathrm{~b}$ and Experiment 2 provided support for Hypothesis 5 , suggesting that in High congruency conditions trust was higher compared to Low congruency conditions (small to medium effects); also, judgments of trust were higher in High congruency conditions relative to the Control conditions (very small to small effects). It is also worth highlighting that in Experiment 2, the only difference between High and Low congruency was the description of the expert (i.e. Academic working group, Government working group). The description of the level of expertise was matched in both groups, so any differences between High and Low congruency conditions regarding judgments of trust were entirely due to attributions that the public made about the fit between expertise and the content of the scientific communication.

In line with Hypothesis 6, in Experiment 1a, 1b, and 2, trust was higher in High congruency compared the Low congruency conditions regardless of whether the behavioral interventions were genuine or not (i.e. plausible vs. implausible) (small to medium effects). In Experiment $1 \mathrm{a}$ and $1 \mathrm{~b}$ the impact of congruency was such that trust was higher in High Congruency conditions for fictitious interventions when compared to trust in Low Congruency conditions for genuine interventions (very small effect). Experiment 2 addressed possible confounds in the details provided to participants by eliminating prestige as a possible factor. The notable aforementioned comparison in Experiment $1 \mathrm{a}$ and $1 \mathrm{~b}$, was not replicated, instead trust was higher in the Low Congruency conditions for genuine interventions, compared to the High Congruency conditions for fictitious interventions (small effect).

Hypothesis 7 was tested in Experiment $1 a$ and $1 b$, for which there was very limited evidential support. There was some impact of conflict of interests on trust judgments overall (very small effects), but the impact was even smaller on judgments of trust as a factor of congruency. Conflicts of interest appeared to have some general impact on ethicality judgments (very small effects). In particular, in the presence of conflicts of interest, fictitious interventions that were opaque were judged highly ethically problematic compared to transparent versions (large effects). In the absence of conflicts of interest, again for fictitious interventions that 
were opaque, ethicality judgments were much harsher than transparent versions (medium effects). By contrast any differences between transparent and opaque nudge for genuine interventions in the presence or absence of conflicts of interest were less pronounced (small effects).

Turning to individual differences, when examined in Experiment $1 \mathrm{a}$ and $1 \mathrm{~b}$, regression analyses revealed that trust in High and Low congruent conditions were much lower in older age groups. The same pattern was found for those that identified themselves as affiliated with very conservative politics, consistent with previous evidence (Gauchat, 2012; Lewandowsky \& Oberauer, 2016). There were few noteworthy sample differences detected in our findings. For plausibility judgments and ethicality judgments the evidence suggested that overall (including both genuine and fictitious intervention) the US judged the behavioral interventions as more plausible and more ethical than the UK sample (very small to small effects). Previous studies that have examined public opinion in European countries have shown that the UK show high approval ratings for a range of nudges and are at comparable to the levels shown in US samples (Reisch, \& Sunstein, 2016). However, Reisch and Sunstein's (2016) survey, and other similar surveys, have not examined judgments of plausibility or ethicality along the lines of the current set of experiments. Therefore, it is hard to infer the extent to which the present findings depart from those of previous surveys.

What is striking is that across both samples, in all three experiments, the basis on which the four judgments reflect differentially sensitivity to three different factors (i.e. the behavioral intervention itself, congruency, conflicts of interest). Perceived transparency judgments tended to be driven by details of the intervention itself. Detailed of potential conflicts of interest tended to impact, to a small degree, judgments regarding trust and ethicality. For plausibility and trust congruency was a salient basis on which judgments were made. In the latter case, although the effects were very small, in Experiment 1 High congruency was enough to inflate judgments of trust to the extent that, even for fictitious nudges, judgments were higher than for genuine nudges proposed in the Low congruency conditions. Does this mean that the credibility of the expert overshadowed attention towards the content of the scientific claims being made? It does appear that congruency effects, as predicted by the Monitoring hypothesis account for the latter finding, but it appears that the public have a far 
more nuanced way of using different cues to inform their attitudes of behavioral interventions designed to target social policy issues.

There is much work in the social sciences suggesting that the public's evaluation of the credibility of scientific claims made by scientists themselves is strongly influenced by factors other than the merit of the specific claims being made. So much so, that the claims can be publicly devalued and easily undermined if attributes of the scientific authority making the claims conflict with those of the individual (e.g. political, gender, social identity) (Hagman et al., 2015; Jung \& Mellers, 2016; Morton, et al., 2006; Nauroth, et al., 2014). In the present set of experiments we show the phenomena operating in a different direction. When looking at the critical differences between the two experimental conditions (High Congruency, Low Congruency), we hypothesised that the likely external factor that influenced judgments regarding the plausibility of the behavioral interventions was the match between the expertise of the source and the topic of the science communication. Clearly the source of the communication is a critical factor (along with the match with the domain of interest being communicated) that boosted judgments directly concerning the expert themselves (judgment of trust), but also judgments which specifically concerned the content of the claims (plausibility judgments) the agents were making.

What is also unusual is that previous psychological research shows that public opinion appears to be more negative with respect to social scientific recommendations that impinge on lifestyle choices (Alemanno, 2012; Diepeveen et al, 2013; Lewandowsky \& Oberauer, 2016). Given the set-up of the present study, public opinion ought to have reflected a similar pattern of results. However, in contrast, the findings from the present set of experiments suggest that, even when behavioral interventions are designed to curb behaviors, the public have a strong positive disposition towards scientists making those proposals over the government. Though one critical difference between previous findings reported here and the present set of experiments is that in the present experiments congruency between source credibility (i.e. expertise) and the topic of social science communication was manipulated.

While our finding presents a positive picture of the trust the public have in scientists in the domain of social policy interventions, it also suggests that the public have a nuanced way of 
assessing behavioral intervention, which involves recruitment of different sources of information that gain relevance depending on the question probing public opinion in surveys. This is certainly important when it comes to attempting to gauge public opinion on matters concerning social scientific policy claims, as well as other scientific findings that inform social policy claims and behavioral interventions such as nudges. In other words, the conclusions that can be drawn regarding positive or negative public attitudes towards scientifically informed social policy communication is going to depend largely on the way in which the questions in the surveys are couched, and the kind of information that be used to assess the credibility of the source of the communication.

Before summing up, we draw the reader's attention to the fact that we used a large sample size in our experiments. What implications does this have for the current research issues we face in the social sciences? The large sample size should give some confidence to the research community that the sample means presented in here are close, that is, they can be trusted as better than usual estimates of corresponding population means (for a formal definition of confidence and close see Trafimow \& MacDonald, 2017, and for a discussion of confidence also see Trafimow, 2017); though a further test of this would be through replication. On this point, again the large sample size should also give the reader reasonable confidence that our findings would also stand up to replication attempts. This is on condition that, as Trafimow (in press) proposes, a successful replication requires that the original study includes a large enough sample size that the sample means are close to the corresponding population means, and the same goes for the replication study. What we hope to highlight here is that our findings, along with our efforts to replicate them, provide the platform for further investigation for other researchers interested in a topic area that is of growing interest to a number of disciplines.

As we discussed at the start of this article, our set of experiments were motived to by a need to better understand public opinion with respect to social policy interventions such as nudges, and to investigate the impact of expertise on public perception of nudges. To this end we show that source credibility is a highly influential factor, but in conjunction with the level of compatibility with the specific domain of scientific research being communicated. So, it is fair to say that our findings show that scientists are more trusted and that this may likely 
influence the more favourable judgments that the public made about the nudges they were presented. But this is largely driven by the fact that there was high compatibility between the expertise and the scientific topic being proposed (actual and perceived). Further work is needed to examine the extent to which the congruency effect is specific to a particular area of science or generalizes to any type of expert and their mapping to the topic they are communicating. Without evidence to the contrary, we would predict that congruency effects of the kind we have shown ought to generalize to any domain of expertise.

In addition, the present experiments were also motivated to contribute to discussions regarding how people navigate the science communication landscape (Strong, 2017). What we show is that the public has a highly nuanced way of navigating science communication, and they rely on different information for different levels of scrutiny and judgment about the communication they are processing. When it comes to ethics, the public care about the potential conflicts of interest of the communicator. When it comes to trust and plausibility of the claims being made, the complement between the communicator and the topic of communication is influential. Taken together these findings have implications for public understanding of science. We show that the public will consider the source, the context that the communication refers to and what is being asked of them as a way to determine their receptiveness of the communication.

\section{Acknowledgments}

This work was supported by the ERC (ERC-2013-AdG339182-BAYES_KNOWLEDGE) 


\section{References}

Agena (2017), 'AgenaRisk Software', http://www.agenarisk

Alemanno, A. (2012), 'Nudging smokers - The behavioural turn of tobacco risk regulation', European Journal of Risk Regulation, 1, 32-42.

Amos, C., Holmes, G., \& Strutton, D. (2008). Exploring the relationship between celebrity endorser effects and advertising effectiveness: A quantitative synthesis of effect size. International Journal of Advertising, 27(2), 209-234.

Arad, A., \& Rubinstein, A. (2015), 'The People's Perspective on Libertarian-Paternalistic

Policies', Unpublished Manuscript. http://arielrubinstein. tau. ac. il/papers/LP. pdf.

Åvitsland, A., Solbraa, A. K., \& Riiser, A. (2017). Promoting workplace stair climbing:

sometimes, not interfering is the best. Archives of public health, 75(1), 2.

Bakeman, R. (2005) Recommended effect size statistics for repeated measure

designs. Behavior Research Methods, 37, 379-384. doi:10.3758/BF03192707;

Barlett, J. E., Kotrlik, J. W., \& Higgins, C. C. (2001), 'Organizational research: Determining appropriate sample size in survey research', Information technology, learning, and performance Journal, 19, 43-50.

BAYES-KNOWLEDGE. (2017), ERC project ERC-2013-AdG339182 $\underline{\text { http://bayes- }}$ knowledge.com/Models/scientific plausibility.cmp.

Benartzi, S., Beshears, J., Milkman, K. L., Sunstein, C. R., Thaler, R. H., Shankar, M., TuckerRay, W., Congdon, W., \& Galing, S. (2017), 'Should Governments Invest More in Nudging?'. Psychological Science, 1-15.

Berman, E. R., \& Johnson, R. K. (2015), 'The unintended consequences of changes in beverage options and the removal of bottled water on a university campus', American journal of public health, 105, 1404-1408.

Brossard, D. (2013). New media landscapes and the science information consumer. Proceedings of the National Academy of Sciences, 110(Supplement 3), 14096-14101.

Botvinick, M. M., Braver, T. S., Barch, D. M., Carter, C. S., \& Cohen, J. D. (2001). Conflict monitoring and cognitive control. Psychological review, 108(3), 624-652.

Cho, M. K., Shohara, R., Schissel, A., \& Rennie, D. (2000). Policies on faculty conflicts of interest at US universities. Jama, 284(17), 2203-2208. 
Critchley, C. R. (2008), 'Public opinion and trust in scientists: The role of the research context, and the perceived motivation of stem cell researchers', Public Understanding of Science, 17, 309-327.

Curchin, K. (2017), 'Using Behavioural Insights to Argue for a Stronger Social Safety Net: Beyond Libertarian Paternalism', Journal of Social Policy, 46, 231-249.

Dana, J., \& Loewenstein, G. (2003). A social science perspective on gifts to physicians from industry. JAMA, 290, 252-255

Diepeveen, S., Ling, T., Suhrcke, M., Roland, M., \& Marteau, T. M. (2013), 'Public acceptability of government intervention to change health-related behaviours: a systematic review and narrative synthesis', BMC Public Health, 13, 1-11.

Drescher, L. S., Roosen, J., \& Marette, S. (2014). The effects of traffic light labels and involvement on consumer choices for food and financial products. International journal of consumer studies, 38(3), 217-227.

Elliott, K. C., \& Resnik, D. B. (2014). Science, policy, and the transparency of values. Environmental health perspectives, 122(7), 647-650.

Ericsson, K. A., \& Lehmann, A. C. (1996). Expert and exceptional performance: Evidence of maximal adaptation to task constraints. Annual review of psychology, 47(1), 273-305.

Felsen, G., Castelo, N., \& Reiner, P. B. (2013), 'Decisional enhancement and autonomy: public attitudes towards overt and covert nudges', Judgment and Decision Making, 8, 202213.

Fenton, N. E. \& Neil, M. (2012), Risk Assessment and Decision Analysis with Bayesian Networks, CRC Press: UK.

Fleck, N., Korchia, M., \& Le Roy, I. (2012). Celebrities in advertising: looking for congruence or likability?. Psychology \& Marketing, 29(9), 651-662.

Gauchat, G. (2012), 'Politicization of Science in the Public Sphere: A Study of Public Trust in the United States, 1974 to 2010', American Sociological Review, 77, 167-187.

Grüne-Yanoff, T., \& Hertwig, R. (2016), 'Nudge versus boost: how coherent are policy and theory?', Minds and Machines, 26, 149-183.

Hagman, W., Andersson, D., Västfjäll, D., Tinghög, G. (2015), 'Public Views on Policies Involving Nudges', Review of Philosophy and Psychology, 6, 439-453.

Hedlin, S., \& Sunstein, C. R. (2016), 'Does Active Choosing Promote Green Energy Use:

Experimental Evidence', Ecology Law Quarterly, 43, 107-142.

Hmielowski, J., Feldman, L., Myers, T., Leiserowitz, A., \& Maibach, E. (2014), 'An attack on science? Media use, trust in scientists, and perceptions of global warming', Public Understanding of Science, 23, 866-883. 
House of Lords. (2011). Science and Technology Select Committee. '2nd Report of Session 2010-12: Behaviour Change' Authority of the House of Lords, London.

Hovland, C., Janis, I., \& Kelley, H. (1953). Communication andpersuasion. New Haven, CT: Yale University Press

Ipsos MORI (2016). 'Politicians are still trusted less than estate agents, journalists and bankers', retrieved from https://www.ipsosmori.com/researchpublications/researcharchive/3685/Politicians-are-still-trusted-lessthan-estate-agents-journalists-and-bankers.aspx [accessed 11 July 2016].

Jarosz, A. F., \& Wiley, J. (2014), 'What are the odds? A practical guide to computing and reporting Bayes factors', The Journal of Problem Solving, 7, 2-9.

Jeffreys, H. (1961), Theory of probability. Oxford: Oxford University Press: UK.

Johnson, E. J., \& Goldstein, D. (2003),'Do defaults save lives?', Science, 302, 1338-1339.

Jung, J. Y., \& Mellers, B. A. M. (2016), 'American attitudes toward nudges', Judgment and Decision Making, 11, 62-74.

Kamins, M. A., \& Gupta, K. (1994). Congruence between spokesperson and product type: A matchup hypothesis perspective. Psychology \& Marketing, 11(6), 569-586.

Kerr, J., Eves, F., \& Carroll, D. (2001). Can posters prompt stair use in a worksite environment?. Journal of Occupational Health, 43(4), 205-207.

Lang, J. T., \& Hallman, W. K. (2005), 'Who does the public trust? The case of genetically modified food in the United States', Risk Analysis, 25, 1241-1252.

Lewandowsky, S., Ecker, U. K., Seifert, C. M., Schwarz, N., \& Cook, J. (2012). Misinformation and its correction: Continued influence and successful debiasing. Psychological Science in the Public Interest, 13(3), 106-131.

Lewandowsky, S., \& Oberauer, K. (2016), 'Motivated rejection of science', Current Directions in Psychological Science, 25, 217-222.

Löfstedt, R. E. (2003). Science communication and the Swedish acrylamide" alarm". Journal of Health Communication, 8(5), 407-432.

Löfstedt, R. (2005). Risk management in post-trust societies. New York: Palgrave Macmillan.

Lupia, A. (2013). Communicating science in politicized environments. Proceedings of the National Academy of Sciences, 110(Supplement 3), 14048-14054.

Marshall, A. L, Bauman, A. E., Patch, C., Wilson, J., \& Chen, J. (2002). Can motivational signs prompt increases in incidental physical activity in an Australian health-care facility? Health Education Research.17, 743-9.

Marques, M. D., Critchley, C. R., \& Walshe, J. (2015), 'Attitudes to genetically modified food over time: How trust in organizations and the media cycle predict support', Public Understanding of Science, 24, 601-618. 
Marteau, T. M. (2017), 'Towards environmentally sustainable human behaviour: targeting non-conscious and conscious processes for effective and acceptable policies', Philosophical Transactions of the Royal Society A, 375, 20160371.

Marteau, T., M., Ogilvie, D., Roland, M., Suhrcke, M., \& Kelly, M. (2011), 'Judging nudging: can nudging improve population health?', British Medical Journal, 342, 263-265.

Mazzocchi, M, Cagnone, S.,Bech-Larsen, T., Niedźwiedzka, B.,Saba, A., Shankar, B., Verbeke, W., \& Traill, B. (2015), 'What is the public appetite for healthy eating policies? Evidence from a cross-European survey', Health Economics, Policy and Law, 10, 3, 267-292.

McComas, K. A. (2008). Session 5: Nutrition communication The role of trust in health communication and the effect of conflicts of interest among scientists: Symposium on 'The challenge of translating nutrition research into public health nutrition'. Proceedings of the Nutrition Society, 67(4), 428-436.

McGuire, W. J. (1978). An information-processing model of advertising effectiveness. In H. L. Davis \& A. J. Silk (Eds.), Behavioral and management sciences in marketing (pp. 156180). New York, NY Wiley.

McKnight, J., \& Coronel, J. C. (2017). Evaluating Scientists as Sources of Science Information: Evidence From Eye Movements. Journal of Communication, 67(4), 565-585.

Michie, S., van Stralen, M. M., \& West, R. (2011), 'The behaviour change wheel: A new method for characterising and designing behaviour change interventions', Implementation Science, 6, 42.

Morton, T. A., Haslam, S. A., Postmes, T., \& Ryan, M. K. (2006), 'We Value What Values Us: The Appeal of Identity-Affirming Science', Political Psychology, 27, 823-838.

Nauroth, P., Gollwitzer, M., Bender, J., \& Rothmund, T. (2014), 'Gamers against science: The case of the violent video games debate', European Journal of Social Psychology, 44, 104-116.

Nisbett, R. E., \& Wilson, T. D. (1977). The halo effect: Evidence for unconscious alteration of judgments. Journal of personality and social psychology, 35(4), 250-256.

Notebaert, W., Gevers, W., Verbruggen, F., \& Liefooghe, B. (2006). Top-down and bottom-up sequential modulations of congruency effects. Psychonomic bulletin \& review, 13(1), 112-117.

Oliver, A. (2013), 'From nudging to budging: using behavioural economics to inform public sector policy', Journal of Social Policy, 42, 685-700.

Oreskes, N., \& Conway, E. M. (2011), Merchants of doubt: How a handful of scientists obscured the truth on issues from tobacco smoke to global warming, Bloomsbury Publishing: USA.

Osman, M. (2016), 'Nudge: How Far Have We Come?', CEconomia. History, Methodology, Philosophy, 6-4, 557-570. 
Petty, R. E., \& Cacioppo, J. T. (1986). The elaboration likelihood model of persuasion. Advances in experimental social psychology, 19, 123-205.

Pornpitakpan, C. (2004). The persuasiveness of source credibility: A critical review of five decades' evidence. Journal of applied social psychology, 34(2), 243-281.

Reisch, L. A., \& Sunstein, C. R. (2016), 'Do Europeans like nudges?', Judgment and Decision Making, 11, 310-325.

Reisch, L., Sunstein, C. R., \& Gwozdz, W. (2016), 'Better Than A Whip? European Attitudes Toward Health Nudges', Food Policy, 69, 1-10.

Rosenstock, L., \& Lee, L. J. (2002). Attacks on science: the risks to evidence-based policy. American Journal of Public Health, 92(1), 14-18.

Sniezek, J. A., \& Van Swol, L. M. (2001). Trust, confidence, and expertise in a judge-advisor system. Organizational behavior and human decision processes, 84(2), 288-307.

Strong, S. I. (2017), 'Alternative Facts and the Post-Truth Society: Meeting the Challenge', University of Pennsylvania Law Review Online, 165, 137-147.

Stroop, J. R. (1935). Studies of interference in serial verbal reactions. Journal of experimental psychology, 18(6), 643-662.

Sturgis, P., \& Allum, N. (2004), 'Science in society: re-evaluating the deficit model of public attitudes',Public understanding of science, 13, 55-74.

Sunstein, C. R. (2014),Simpler: The future of government. Simon and Schuster: USA.

Sunstein, C. R. (2016a). The ethics of influence: Government in the age of behavioral science. Cambridge University Press: USA.

Sunstein, C. R. (2016b), 'People prefer system 2 nudges (kind of)', Duke Law Journal, 66, 121168.

Sunstein, C. R., Reisch, L. A., \& Rauber, J. (2017), 'A World-Wide Consensus on Nudging? Not Quite, But Almost' SSRN https://ssrn.com/abstract=2955693. http://dx.doi.org/10.2139/ssrn.2955693

Teeny, J., Briñol, P., \& Petty, R. E. (2016). The elaboration likelihood model. Routledge International Handbook of Consumer Psychology, 390.

Thorndike, E. L A constant error in psychological ratings. Journal of Applied Psychology, 1920, 4, 25-29.

Trafimow, D. (in press). An A Priori Solution to the Replication Crisis. Philosophical Psychology.

Trafimow, D. (2017). Using the coefficient of confidence to make the philosophical switch from a posteriori to a priori inferential statistics. Educational and Psychological Measurement, 77, 831-854. 
Trafimow, D., \& MacDonald, J. A. (2017). Performing inferential statistics prior to data collection. Educational and Psychological Measurement, 77, 204-219.

Wagenmakers, E. J. (2007), 'A practical solution to the pervasive problems of $p$ values', Psychonomic Bulletin \& Review, 14, 779-804.

Winter, S., \& Krämer, N. C. (2012). Selecting science information in Web 2.0: How source cues, message sidedness, and need for cognition influence users' exposure to blog posts. Journal of Computer-Mediated Communication, 18(1), 80-96. 